\title{
ON A PROBLEM OF GRONWALL FOR BAZILEVIČ FUNCTIONS
}

\author{
BY
}

JOHN L. LEWIS

ABSTRACT. Let $B(a, \beta)$, a positive, $\beta$ real, denote the class of normalized univalent Bazilevič functions in $K=\{z:|z|<1\}$ of type $a, \beta$. Let $B=$ $\cup_{a, \beta} B(a, \beta)$. Let $a, 0 \leq a \leq 2$, and $a, 0<a<\infty$, be fixed and suppose that $f(z)=z+a z^{2}+\cdots$ is in $B(a, 0)$. In this paper for given $z_{0} \in K$, the author finds a sharp upper bound for $\left|f\left(z_{0}\right)\right|$. Also, a sharp asymptotic bound is obtained for $(1-r)^{2} \max |z|=r|f(z)|$. Finally, a sharp asymptotic bound is found for $(1-r)^{2} \max |z| \Rightarrow r|f(z)|$ when $f$ is in $B$ with second coefficient $a$.

1. Introduction. 'Let $S$ denote the class of univalent functions $f$ in $K=$ $\{z:|z|<1\}$ with the normalization $f(0)=0, f^{\prime}(0)=1$. Let $M(r, f)=\max |z|=r|f(z)|$, $0<r<1$. Then in [3] Gronwall proposed the following problem.

Problem 1. Given $a, 0 \leq a \leq 2$, and $r_{0}, 0<r_{0}<1$, if $f(z)=z+\sum_{n=2}^{\infty} a_{n} z^{n}$ is in $S$ with $\left|a_{2}\right|=a$, find the maximum of $M\left(r_{0}, f\right)$.

In [6] Jenkins solved Problem 1 using quadratic differentials. The maximum is attained by a unique function $G=G\left(\cdot, a, r_{0}\right)$ in $S$ with second coefficient $a$ and $M\left(r_{0}, G\right)=G\left(r_{0}, a, r_{0}\right)$. $G$ maps $K$ onto a region whose complement is a forked slit, symmetric about the real axis. Depending on $a$ and $r_{0}$, the handle of the fork is either (a) on the negative real axis, (b) at $\propto$, or (c) along the positive real axis. As $r_{0} \rightarrow 1$ Jenkins showed

$$
\left(1-r_{0}\right)^{2} M\left(r_{0}, G\right) \rightarrow 4 b^{-2} \exp \left(2-4 b^{-1}\right), \quad b=2-(2-a)^{1 / 2}
$$

Let $f$ be as in Problem 1. Then Hayman (see [4, Theorem 1.5]) has shown that $r^{-1}(1-r)^{2} M(r, f)$ is a decreasing function of $r, 0<r<1$. It follows from this fact and (1.1) that

$$
\lim _{r \rightarrow 1}(1-r)^{2} M(r, f) \leq 4 b^{-2} \exp \left(2-4 b^{-1}\right), \quad b=2-(2-a)^{1 / 2},
$$

with equality holding for some $f$ in $S$ with $\left|a_{2}\right|=a$.

Received by the editors March 13, 1973 and, in revised form, September 16, 1973. AMS (MOS) subject classifications (1970). Primary 30A32, 30A40.

Key words and phrases. Univalent functions, Bazilevič functions, extremal functions, asymptotic growth. 
In this paper we consider Problem 1 in certain classes of univalent functions. More specifically, for given $\alpha, 0<\alpha<\infty$, and $\beta,-\infty<\beta<\infty$, let $B(\alpha, \beta)$ denote the normalized Bazilevic functions of type $\alpha, \beta$ (see [1]). That is, $f \in B(\alpha, \beta)$ if $f(0)=0, f^{\prime}(0)=1$, and

$$
f(z)=z\left[(\alpha+i \beta) z^{-(a+i \beta)} \int_{0}^{z}\left(\frac{\phi(\zeta)}{\zeta}\right)^{a} P(\zeta) \zeta^{(a+i \beta-1)} d \zeta\right]^{1 /(a+i \beta)}
$$

where $z \in K-\{0\}, \phi$ is starlike univalent with $\phi(0)=0,\left|\phi^{\prime}(0)\right|=1$, and $P$ has positive real part with $|P(0)|=1$. The symbol $(\phi(\zeta) / \zeta)^{a}$ denotes an analytic $a$ power of $\phi(\zeta) / \zeta$ in $K$. All other powers inside the brackets are principal values, and the integral is taken along the line segment from 0 to $z$. The power outside the brackets is the analytic $(\alpha+i \beta)^{-1}$ power of the function inside the brackets which approaches 1 as $z \rightarrow 0$. We note that $B(1,0)$ is the class of normalized close to convex functions. We put $B=\bigcup_{\alpha, \beta} B(\alpha, \beta)$ and call $f \in B$ a Bazilevic function.

Functions $f \in B$ have been shown to be univalent by Bazilevič (see also [7], [9]). They are the largest class of univalent functions given by an explicit representation formula. We remark that the extremal functions $G\left(\cdot, a, r_{0}\right)$ with image domains as in (a) or (c) are not Bazilevič. This can be seen by using a geometric characterization of $B(\alpha, \beta)$ due to Sheil-Small (see [9, Theorem 2]). Therefore it is worthwhile to consider Problem 1 in $B$. To do this we first suppose that $a$ is fixed, $0<a<\alpha$, and $\beta=0$.

For given $a, 0 \leq a \leq 2$, we introduce the functions $F(\cdot, \sigma), 1 \leq \sigma \leq 2 a+1$, defined by

$$
F(z, \sigma)=\left\{\alpha \int_{0}^{z} \frac{\zeta^{a-1}\left(1+2 c \zeta+\zeta^{2}\right)}{(1-\zeta)^{2 a+2-\sigma}(1+\zeta)^{\sigma}} d \zeta\right\}^{1 / a}
$$

where $z \in K$ and

$$
2 c=(\alpha+1) a-2 \alpha-2+2 \sigma .
$$

Let

$$
r_{1}=\max \{a-1 / 2(\alpha+1) a, 1\}, \quad r_{2}=\max \{\alpha+1-1 / 2(\alpha+1) a, 1\} \text {. }
$$

For $\tau_{1} \leq \sigma \leq \tau_{2}$ we observe that $F(., \sigma)$ is in $B(a, 0)$ and $F(z, \sigma)=z+a z^{2}+\cdots$ Then we shall prove

Theorem 1. Let $a$ and $r_{0}$ be fixed numbers where $0 \leq a \leq 2$ and $0<r_{0}<1$. Let $f(z)=z+\sum_{n=2}^{\infty} a_{n} z^{n}$ be in $B(\alpha, 0)$ with $\left|a_{2}\right|=a$. Then 


$$
M\left(r_{0}, f\right) \leq \max _{r_{1} \leq \sigma \leq r_{2}} F\left(r_{0}, \sigma\right)
$$

If $a \geq 2 \alpha /(\alpha+1)$, then $r_{2}=1$ and $M\left(r_{0}, f\right) \leq F\left(r_{0}, 1\right)$. Equality bolds if and only if for some real $\theta, f(z)=e^{-i \theta} F\left(e^{i \theta} z, 1\right), z \in K$. As $r \rightarrow 1$,

$$
\lim _{r \rightarrow 1}(1-r)^{2} M(r, f) \leq \xi(\alpha)=[\max \{0,1 / 4(2(1-a)+(\alpha+1) a)\}]^{1 / a} .
$$

If $\xi(\alpha)>0$, then equality holds if and only if for some real $\theta$ we have $f(z)=$ $e^{-i \theta} F\left(e^{i \theta} z, 1\right), z \in K$.

From Theorem 1 we see that if $a \geq 2 a /(\alpha+1)$, then the maximum in (1.7) is attained by $F(., 1)$ independent of $r_{0}$. However if $0 \leq a<2 a /(\alpha+1)$, then it follows from our proof (see (2.4)) that for $r_{0}$ near zero the maximum in (1.7) is attained only for $\sigma$ near $\tau_{2}$, while for $r_{0}$ near 1 the maximum is attained only for $\sigma$ near $r_{1}\left(r_{1}<r_{2}\right)$. In general for a fixed $r_{0}$ and $a$, the question of whether the maximum in (1.7) occurs for exactly one value of $\sigma$ is still open.

Most of the proof of Theorem $1(\$ 2)$ is based upon simple properties of subordination and the observation that it suffices to consider functions with real coefficients. Similar properties of subordination have been used by Gronwall [3] and Finkelstein [2] to solve Problem 1 for starlike univalent functions. However, we must use a more difficult argument to prove uniqueness in $(1.8)$ for $\xi(\alpha)>0$ (see $\$ 3)$.

In $\$ 4$ we first show if $f \in B(\alpha, \beta)$ and $\beta \neq 0$, then $M(r, f)=o\left[(1-r)^{-2}\right]$ as $r \rightarrow 1$. We then obtain easily from Theorem 1:

Theorem 2. Let a be fixed, $0 \leq a \leq 2$. Let $f(z)=z+\sum_{n=2}^{\infty} a_{n} z^{n}$ be in $B$ with $\left|a_{2}\right|=a$. Then

$$
\lim _{r \rightarrow 1}(1-r)^{2} M(r, f) \leq \max _{0<a<\infty} \xi(\alpha)=\xi\left(a_{0}\right)
$$

where $a_{0}$ is the root of the equation

$$
a(a-2)-[2(1-a)+a(1+\alpha)] \log \left[\frac{1}{4}(2(1-\alpha)+a(1+\alpha))\right]=0 .
$$

Equality bolds only if for some real $\theta, f(z)=e^{-i \theta} F\left(e^{i \theta} z, 1\right), z \in K$, where $F(., 1)$ is defined as in (1.4) with $a=a_{0}$.

We remark that (1.10) implies $\alpha_{0}$, for $0 \leq a<2$, is an increasing function of $a$ and $a_{0} \rightarrow+\infty$ as $a \rightarrow 2$. Also, we remark that the extremal function $F(., 1)$ in Theorem 2 for $0 \leq a<2$ is in $B(\alpha, \beta)$ only when $\alpha=a_{0}$ and $\beta=0$, since otherwise it could not be extremal. Finally we remark for $f$ as in Theorem 2 that $\lim _{n \rightarrow \infty} a_{n} / n \leq \xi\left(a_{0}\right)$. This inequality follows directly from Theorem 2 and a theorem of Hayman (see [4, Theorem 5.7]). 
In $\$ 5$ we compare the bound given by (1.9) with the bound given by (1.2) for different values of $a$. We also discuss mapping properties of the function $F(., 1)$ for $a=1 / 2,1,2$, and $0 \leq a<2$ in this section.

2. Proof of Theorem 1. The proof of Theorem 1 is based upon the following lemmas (see Finkelstein [2] for similar lemmas).

Lemma 1. Let $\omega$ be analytic in $K$ with $|\omega(z)| \leq 1, z \in K, \omega(0)=0$ and $\omega^{\prime}(0)=b,-1 \leq b \leq 1$. If $\omega(r)$ is real, $0<r<1$, then $\omega(r) \leq r(r+b) /(1+r b)$, $0<r<1$, with equality bolding only for $\omega(z)=z(z+b) /(1+b z), z \in K$.

Proof. Apply Schwarz's lemma to the function $[\omega(z)-b z V / z-b \omega(z)], z \in K$.

Lemma 2. Let $Q$ be an analytic function in $K$ with positive real part and $Q(0)=1, Q^{\prime}(0)=2 b,-1 \leq b \leq 1$. If $Q(r)$ is real for $0<r<1$, then

$$
Q(r) \leq\left(1+2 b r+r^{2}\right) /\left(1-r^{2}\right), \quad 0<r<1 .
$$

Equality holds only for $Q(z)=\left(1+2 b z+z^{2}\right) /\left(1-z^{2}\right), z \in K$.

Proof. From the hypotheses on $Q$ we have $Q=(1+\omega) /(1-\omega)$, where $\omega$ is as in Lemma 1. Applying Lemma 1 and using the fact that the function $x \rightarrow$ $(1+x) /(1-x)$ is increasing for $-1 \leq x<1$, we get Lemma 2 .

Lemma 3. Let $\psi$ be starlike univalent in $K$ with $\psi(0)=0, \psi^{\prime}(0)=1$, and $\psi^{\prime \prime}(0)=4 b,-1 \leq b \leq 1$. If $\psi(r)$ is real, $0<r<1$, then

$$
\psi(r) \leq r(1-r)^{-1-b}(1+r)^{b-1}, \quad 0<r<1
$$

Equality bolds only for $\psi(z)=z(1-z)^{-1-b}(1+z)^{b-1}$.

Proof. From the hypotheses on $\psi$ we have $z \psi^{\prime}(z) / \psi(z)=Q(z)$, where $Q$ is as in Lemma 2. Applying Lemma 2 and integrating we get Lemma 3.

We now prove (1.7) of Theorem 1. Let $a$ and $r_{0}$ be fixed numbers where $0 \leq a \leq 2$ and $0<r_{0}<1$. Let $f \in B(a, 0)$ and $f(z)=z+\sum_{n=2}^{\infty} a_{n} z^{n}$ with $\left|a_{2}\right|=a_{0}$. By making a rotation, if necessary, we may assume that $\left|f\left(r_{0}\right)\right|=M\left(r_{0}, f\right)$. Next suppose that $f$ has the form (1.3) with $\beta=0$, where $\phi$ and $P$ are as defined there. We put

$$
\begin{gathered}
\psi(z)=z\left[\frac{\phi(z)}{z} \cdot \frac{\overline{\phi(\bar{z})}}{z}\right]^{1 / 2}, \quad Q(z)=[P(z) \cdot \overline{P(\bar{z})}]^{1 / z}, \\
g(z)=z\left[\alpha z^{-a} \int_{0}^{z}\left(\frac{\psi(\zeta)}{\zeta}\right)^{a} Q(\zeta) \zeta^{a-1} d \zeta\right]^{1 / \alpha},
\end{gathered}
$$


when $z \in K$. Here the analytic $1 / 2$ powers are 1 at $z=0$. It is easily checked that $\psi$ is starlike univalent with $\psi(0)=0, \psi^{\prime}(0)=1$, and that $Q$ has positive real part with $Q(0)=1$. From this fact we see that $g \in B(\alpha, 0)$ provided the powers in the expression for $g$ are given the same interpretation as in (1.3). Since $\psi(r)=|\phi(r)|$ and $Q(r)=|P(r)|, 0 \leq r<1$, we have the inequality

$$
M\left(r_{0}, f\right) \leq g\left(r_{0}\right)
$$

Also, $g(z)=z+\operatorname{Re} a_{2} z^{2}+\cdots$ and $Q, \psi$, have real coefficients.

From the above remarks we see that $Q$ and $\psi$ satisfy the hypotheses of Lemmas 2 and 3. Applying these lemmas we obtain for some $\sigma$ that

$$
g\left(r_{0}\right)^{a} \leq \alpha \int_{0}^{r_{0}} \frac{\left(1+s^{2}+2 c_{0} s\right) s^{2-1} d s}{(1-s)^{2 a+2-\sigma}(1+s)^{\sigma}}
$$

where $2 c_{0}=(a+1) \operatorname{Re} a_{2}+2 \sigma-2 a-2,1 \leq \sigma \leq 2 a+1$, and $-1 \leq c_{0} \leq 1$. Let $F(\cdot, \sigma)$ and $c$ be as in (1.4) and (1.5). Then since $c_{0} \leq c$, it follows from (2.1) and (2.2) that

$$
M\left(r_{0}, f\right) \leq g\left(r_{0}\right) \leq F\left(r_{0}, \sigma\right)
$$

Let $r_{1}$ and $r_{2}$ be as in (1.6). Then if $\sigma>r_{2}$, we consider $F\left(r_{0},.\right)$ as a function of $\sigma$ for $r_{2}<\sigma \leq 2 a+1$. We obtain since $c>0$ that

$$
\begin{aligned}
& \frac{\partial F^{a}}{\partial \sigma}\left(r_{0}, \sigma\right) \\
& \quad=a \int_{0}^{r_{0}} \frac{\left(1+s^{2}+2 c s\right) s^{a-1}}{(1-s)^{2 a+2-\sigma}(1+s)^{\sigma}}\left[\frac{2 s}{\left(1+s^{2}+2 c s\right)}+\log \left(\frac{1-s}{1+s}\right)\right] d s<0,
\end{aligned}
$$

for $\tau_{2}<\sigma \leq 2 a+1$. Hence $F\left(r_{0}, \sigma\right)<F\left(r_{0}, \tau_{2}\right), \tau_{2}<\sigma \leq 2 a+1$.

If $1 \leq \sigma<r_{1}$, then $c_{0} \leq c<-1$, which is a contradiction to the fact that $-1 \leq c_{0} \leq 1$. We conclude that (1.7) is true.

If $a \geq 2 a /(\alpha+1)$, then $r_{2}=1$, and from (1.7) we have $M\left(r_{0}, f\right) \leq F\left(r_{0}, 1\right)$. From Lemmas $1-3$ and (2.4), we deduce that equality can hold only for rotations of $F(., 1)$.

Next we prove (1.8). First suppose that $a-(\alpha+1) a / 2<1$. Then from (1.5) we see that $c>(a+1) a / 2-a>-1$ for $r_{1}=1 \leq \sigma \leq \tau_{2}$. It follows from this inequality that the integrand in (2.4) is negative on $\left[s_{0}, 1\right)$ provided $s_{0}$ is near enough 1. Also the choice of $s_{0}$ does not depend on $\sigma, 1 \leq \sigma \leq \tau_{2}$. Hence there 
exists a positive constant, $A$, which does not depend on $\sigma$, such that $\max _{1 \leq \sigma \leq r_{2}} F(r, \sigma) \leq A+F(r, 1), 0<r<1$. Using this inequality, (1.7), and l'Hospital's rule we get

$$
\begin{aligned}
\lim _{r \rightarrow 1}(1-r)^{2} M(r, f) & \leq \lim _{r \rightarrow 1}(1-r)^{2} F(r, 1)=\left[\lim _{r \rightarrow 1}(1-r)^{2 a} F^{a}(r, 1)\right]^{v / a} \\
& =\left[\lim _{r \rightarrow 1}(2 a)^{-1}(1-r)^{2 a+1} \frac{d}{d r} F^{a}(r, 1)\right]^{1 / a}=\xi(\alpha) .
\end{aligned}
$$

If $a-(\alpha+1) a / 2 \geq 1$, we note for fixed $\sigma$ and $r_{0}$ that the value of $F\left(r_{0}, \sigma\right)$ increases with $a$, as follows from (1.5). Next, given $a_{1}, 2 \geq a_{1}>2(\alpha-1) /(a+1)$ we replace $a$ by $a_{1}$ in (1.4). We obtain for fixed $\sigma, 1 \leq \sigma \leq 2 a+1$, a new function $G=G(\cdot, \sigma)$ in $K$. Furthermore, from the above remark and (2.3) it is clear that $M\left(r_{0}, f\right) \leq F\left(r_{0}, \sigma\right) \leq G\left(r_{0}, \sigma\right)$ where $1 \leq \sigma \leq 2 a+1$. Using this inequality and arguing as in the proof of (2.5) we find that

$$
\lim _{r \rightarrow 1}(1-r)^{2} M(r, f) \leq\left[\max \left\{0,1 / 4\left(2(1-a)+(a+1) a_{1}\right)\right\}\right]^{1 / a}
$$

Letting $a_{1} \rightarrow 2(a-1) /(\alpha+1)$, we get $\lim _{r \rightarrow 1}(1-r)^{2} M(r, f)=0$. Hence $(1.8)$ is true.

3. A uniqueness proof. It remains to show for $\xi(\alpha)>0$ that equality holds in (1.8) only for $f(z)=e^{-i \theta} F\left(e^{i \theta} z, 1\right), z \in K, \theta$ real. This can be shown by the following argument. Suppose that $\lim _{r \rightarrow 1}(1-r)^{2} M(r, f)=\xi(a)$. Then by Hay$\operatorname{man}($ see $[4$, Theorem 5.6]) there exists $\psi, 0 \leq \psi<2 \pi$, such that

$$
\lim _{r \rightarrow 1}(1-r)^{2}\left|f\left(r e^{i \psi}\right)\right|=\xi(\alpha)
$$

By making a rotation if necessary, we may assume that $\psi=0$. We recall that $f$ has the form (1.3) with $\beta=0$, where $\phi$ and $P$ are as defined there. We claim that

$$
\phi(z)=e^{i \tau} z /(1-z)^{2}, \quad z \in K,
$$

for some real $\tau$. Indeed, by a theorem of Pommerenke [8] either $\phi(z)=$ $e^{i \tau} z /\left(1-e^{i \gamma} z\right)^{2}$ for some real $r$ and $\gamma$ or $M(r, \phi)=o\left[(1-r)^{-2}\right]$. The second possibility cannot occur, since it would imply $\xi(\alpha)=0$. From (3.1) the first possibility can occur only if $\gamma=0$. Hence our claim is true.

Next we note that $f(z)=z+a z^{2}+\cdots$. Otherwise defining $g$ as previously and arguing as in $\$ 2$, we could obtain a contradiction to (3.1). It follows from this fact and (3.2) that 


$$
P(z)=e^{-i a r}(1+2 c z+\cdots)
$$

where $c$ is as in (1.5) with $\sigma=1$. To complete the proof of uniqueness we show that $e^{i \bar{a} r}=1$ and

$$
P(z)=\left(1+2 c z+z^{2}\right) /\left(1-z^{2}\right), \quad z \in K
$$

First from the Herglotz representation formula we see that $P$ may be written in the form

$$
P(z)=\cos \alpha r \int_{-\pi}^{\pi}\left(\frac{1+e^{-i \theta} z}{1-e^{-i \theta} z}\right) d y(\theta)-i \sin \alpha r, \quad z \in K,
$$

where $\gamma$ is a nondecreasing function on $[-\pi, \pi]$ which is continuous on the right and $\gamma(\pi)-\gamma(-\pi)=1$. Second from Theorem 2 of Hayman [5] and its subsequent proof we see that

$$
\lim _{r \rightarrow 1} \frac{1-r}{1+r} P(r)=\cos \operatorname{ar}\left[\gamma\left(0^{+}\right)-\gamma\left(0^{-}\right)\right]=\lambda .
$$

Using (3.1) and (3.2) it follows that

$$
\begin{aligned}
(1-r)^{2 a}|f(r)|^{a} & =\lambda a(1-r)^{2 a} \int_{0}^{r} \frac{\rho^{a-1}(1+\rho)}{(1-\rho)^{2 a+1}} d \rho+o(1) \\
& =\lambda r^{a}+o(1) \rightarrow \xi^{a}(a)=1 / 2(1+c),
\end{aligned}
$$

as $r \rightarrow 1$. Hence,

$$
\lambda=(1+c) / 2
$$

Let $\gamma_{1}(\theta)=0$ for $-\pi \leq \theta<0$ and $\gamma_{1}(\theta)=\gamma\left(0^{+}\right)-\gamma\left(0^{-}\right)$for $0 \leq \theta \leq \pi$. Then since $\gamma$ is continuous on the right, the function $\gamma_{2}=\gamma-\gamma_{1}$ is increasing on $[-\pi, \pi]$. Using this fact and (3.5)-(3.7) it follows that

$$
H(z)=P(z)-\frac{(1+c)}{2}\left(\frac{1+z}{1-z}\right)+i \sin \text { ar }=\cos a r \int_{-\pi}^{\pi}\left(\frac{1+e^{-i \theta} z}{1-e^{-i \theta} z}\right) d \gamma_{2}(\theta)
$$

has positive real part and $\cos a r\left[\gamma_{2}(\pi)-\gamma_{2}(-\pi)\right]=\cos a r-(1+c) / 2$. Using (3.3) and comparing coefficients in the above formula for $H$, we get

$$
\begin{aligned}
H(z) & =\cos \alpha r-1 / 2(1+c)+\left[2 c e^{-i \alpha r}-(1+c)\right] z+\cdots \\
& =\cos \alpha r-1 / 2(1+c)+2 \cos \alpha_{r}\left(\int_{-\pi}^{\pi} e^{-i \theta} d \gamma_{2}(\theta)\right) z+\cdots
\end{aligned}
$$

Hence 


$$
\begin{aligned}
{[2 c \cos \alpha r-(1+c)] } & =2 \cos \alpha r \int_{-\pi}^{\pi} \cos \theta d \gamma_{2}(\theta) \\
& \geq-2 \cos \alpha r\left[\gamma_{2}(\pi)-\gamma_{2}(-\pi)\right]=-2 \cos \alpha r+(1+c),
\end{aligned}
$$

where equality holds only if $\gamma_{2}$ is constant on $[-\pi, \pi)$. Since $-1<c \leq 1$, the above inequality implies that $\cos a r \geq 1$. Hence $\gamma_{2}$ is constant on $[-\pi, \pi)$ and $\cos a r=1$. We conclude that

$$
H(z)=1 / 2(1-c)(1-z) /(1+z), \quad z \in K,
$$

and thereupon that (3.4) is true. The proof of uniqueness is now complete.

4. Proof of Theorem 2. We begin the proof of Theorem 2 by showing that if $f \in B(\alpha, \beta)$ and

$$
\lim _{r \rightarrow 1}(1-r)^{2} M(r, f)>0
$$

then $\beta=0 .(4.1)$ implies, as in $\$ 3$, that

$$
\lim _{r \rightarrow 1}(1-r)^{2}\left|f\left(r e^{i \psi}\right)\right|>0
$$

for some real $\psi$. Again we may assume $\psi=0$. We observe $(1+r)^{-1}(1-r)^{3}\left|f^{\prime}(r)\right|$ is a decreasing function of $r$ for $0<r<1$, as follows easily from the well-known inequality,

$$
(\partial / \partial \rho) \log \left|f^{\prime}(\rho)\right| \leq(2 \rho+4) /\left(1-\rho^{2}\right), \quad 0<\rho<1 .
$$

(For a proof see Hayman $\left[4\right.$, p. 5].) Also, $|f(r)| \leq \int_{0}^{r}\left|f^{\prime}(\rho)\right| d \rho$. Using, these ob. servations we deduce that

$$
\lim _{r \rightarrow 1}(1-r)^{3}\left|f^{\prime}(r)\right|>0
$$

since otherwise we could contradict (4.2).

Before proceeding further we adopt the following notation. If $b$ is analytic in $K, b(z) \neq 0, z \in K$, and $b(0)=1$, we let $\log b$ denote the analytic logarithm of $b$ in $K$ for which $\log b(0)=0$. Furthermore we let $\arg b(z)=\operatorname{Im} \log b(z)$. In terms of this notation we have

$$
\begin{aligned}
\left|\log r^{-1} f(r)\right| \leq-2(1+o(1)) \log (1-r), & r \rightarrow 1, \\
\left|\log f^{\prime}(r)\right| \leq-3(1+o(1)) \log (1-r), & r \rightarrow 1 .
\end{aligned}
$$


(4.4) is a simple consequence of the well-known inequality

$$
\left|\frac{f^{\prime}(z)}{f(z)}\right| \leq \frac{1+\rho}{\rho(1-\rho)}, \quad|z|=\rho, 0<\rho<1,
$$

and (4.5) is a consequence of the inequality

$$
\left|\frac{z f^{\prime \prime}(z)}{f^{\prime}(z)}-\frac{2 \rho^{2}}{\left(1-\rho^{2}\right)}\right| \leq \frac{4 \rho}{\left(1-\rho^{2}\right)}, \quad|z|=\rho, 0<\rho<1
$$

(see Hayman $[4$, p. 5] for inequalities (4.4a), (4.5a)).

For completeness we give the proof of (4.5). From (4.5a) we see that

$$
\left|\frac{f^{\prime \prime}(z)}{f^{\prime}(z)}\right| \leq \frac{4+2 \rho}{\left(1-\rho^{2}\right)}, \quad|z|=\rho, 0<\rho<1 .
$$

Using this inequality we obtain

$$
\begin{aligned}
\left|\log f^{\prime}(r)\right| & =\left|\int_{0}^{r} \frac{f^{\prime \prime}(\rho)}{f^{\prime}(\rho)} d \rho\right| \leq \int_{0}^{r}\left|\frac{f^{\prime \prime}(\rho)}{f^{\prime}(\rho)}\right| d \rho \leq \int_{0}^{r} \frac{4+2 \rho}{\left(1-\rho^{2}\right)} d \rho \\
& =\log (1+r)-3 \log (1-r) .
\end{aligned}
$$

From (4.2) and (4.4) it is easily seen that

$$
\arg \frac{f(r)}{r}=-o(1) \log (1-r), \quad r \rightarrow 1
$$

and from (4.3) and (4.5) that

$$
\arg f^{\prime}(r)=-o(1) \log (1-r), \quad r \rightarrow 1 .
$$

Next we note that

$$
(\alpha+i \beta-1) \log z^{-1} f(z)+\log f^{\prime}(z)=\log \left[\left(z^{-1} \phi(z)\right)^{a} P(z)\right]
$$

(4.8) is a consequence of the representation formula (1.3) for f. Using (4.6), (4.7), (4.2), and taking imaginary parts in (4.8) for $z=r$, we get

$$
(\beta+o(1)) \log \left|r^{-1} f(r)\right|=-2(\beta+o(1)) \log (1-r)=\arg \left[\left(r^{-1} \phi(r)\right)^{a} \circ P(r)\right] .
$$

The right-hand side of this equality remains bounded as $r \rightarrow 1$, since $\phi$ is starlike univalent and $P$ has positive real part. Hence we must have $\beta=0$ and $f \in B(\alpha, 0)$.

We now prove Theorem 2. From the above argument we may assume $f \in B(\alpha, 0)$ 
and $f(z)=z+\sum_{n=0}^{\infty} a_{n} z^{n}$, with $\left|a_{2}\right|=a$. Then from Theorem 1 it follows that

$$
\lim _{r \rightarrow 1}(1-r)^{2} M(r, f) \leq \max _{0<a<\infty} \xi(\alpha)=\xi\left(a_{0}\right)
$$

Using the differential calculus we deduce for $0 \leq a<2$ that $a_{0}$ is the unique root of equation (1.10).

5. Some remarks on Theorem 2. The following table gives a comparison between the bound in (1.2) for $T(a)=4 b^{-2} \exp \left(2-4 b^{-1}\right), b=2-(2-a)^{1 / 2}$, and the bound $\xi\left(a_{0}\right)$ in (1.9) for different values of $a$. From the table it can be seen that the maximum difference is about .032 .

$\begin{array}{cccc}a & a_{0} & \xi\left(a_{0}\right) & T(a) \\ 0 & .63 & .069 & .093 \\ .2 & .74 & .127 & .157 \\ .4 & .87 & .205 & .237 \\ .6 & 1.03 & .300 & .331 \\ .8 & 1.22 & .407 & .434 \\ 1.0 & 1.47 & .520 & .541 \\ 1.2 & 1.81 & .634 & .649 \\ 1.4 & 2.30 & .743 & .752 \\ 1.6 & 3.12 & .843 & .848 \\ 1.8 & 4.98 & .931 & .933 \\ 1.9 & 7.6 & .968 & .969 \\ 1.99 & 26.9 & .997 & .997\end{array}$

We now discuss the mapping properties of $F(\cdot, 1)$. If $a=2$ and $0<a<\infty$, then $F(z, 1)=z /(1-z)^{2}, z \in K$. In general for a fixed $a, 0<a<\infty$, and $a, 0 \leq a$ $<2$, the image domain corresponding to $K$ under the mapping $F(\cdot, 1)$ does not have a simple geometric description. Therefore we consider only the cases $\alpha=$ $1 / 2,1,2$, and $0 \leq a<2$, where such a description is possible. If $0 \leq a<2$ and $c$ is as in (1.5) with $\sigma=1$, we let

$$
\gamma=a \int_{0}^{e^{i \theta}} \frac{z^{a-1}}{(1-z)^{2 a+1}} \frac{\left(1+2 c z+z^{2}\right)}{(1+z)} d z, \quad z \in K
$$

where $\cos \theta=-c$ and $0<\theta<\pi$.

First, if $a=1 / 2$, and $0 \leq a<2$, then the function $F^{1 / 2}(z, 1)$ is univalent in $D=K \cap\{z: \operatorname{Im} z>0\}$. Here the square root is intended as a principal value. Let 
$\Omega=F^{1 / 2}(D, 1)$. Then the boundary of $\Omega$ consists of the line segments:

$$
\begin{aligned}
& l_{1}=\{w: \operatorname{Re} w \geq 0, \operatorname{Im} w=0\}, \quad l_{2}=\{w: \operatorname{Im} w \geq 0, \operatorname{Re} w=0\}, \\
& l_{3}=\{w: \operatorname{Re} w=\operatorname{Re} \gamma, \operatorname{Im} w \geq \operatorname{Im} \gamma\}
\end{aligned}
$$

as we find from examining the tangent to the boundary. It follows that $F(\cdot, 1)$ maps $D$ onto the region in the $w=u+i v$ plane whose complement is the closure of the lower half plane and the parabolic arc

$$
u=(\operatorname{Re} \gamma)^{2}-(v / 2 \operatorname{Re} \gamma)^{2}, \quad v \geq 2 \operatorname{Im} \gamma \operatorname{Re} \gamma .
$$

Using the reflection principle we deduce that $F(., 1)$ maps $K$ onto the region whose complement is the parabolic arcs,

$$
\begin{array}{ll}
u=(\operatorname{Re} \gamma)^{2}-(v / 2 \operatorname{Re} \gamma)^{2}, & v \geq 2 \operatorname{Im} \gamma \operatorname{Re} \gamma \\
u=(\operatorname{Re} \gamma)^{2}-(v / 2 \operatorname{Re} \gamma)^{2}, & v \leq-2 \operatorname{Im} \gamma \operatorname{Re} \gamma .
\end{array}
$$

As $a \rightarrow 2$ the parabolic arcs approach the line segment $(-\infty,-1 / 4]$.

Second if $a=1$, and $0 \leq a<2$, then $F$ maps $K$ onto the region whose complement is the line segments:

$\operatorname{Re} u \leq \operatorname{Re} \gamma, \quad \operatorname{Im} u=\operatorname{Im} \gamma, \quad \operatorname{Re} u \leq \operatorname{Re} \gamma, \operatorname{Im} u=-\operatorname{Im} \gamma$.

Third, if $a=2$, then from an analysis similar to the case $\alpha=1 / 2$, we find that $F(., 1)$ maps $K$ onto a region whose complement is the hyperbolic arcs:

$$
\begin{array}{lll}
2 u v=\operatorname{Im} \gamma, & u^{2}-v^{2} \geq \operatorname{Re} \gamma, & v \geq 0, \\
2 u v=-\operatorname{Im} \gamma, & u^{2}-v^{2} \geq \operatorname{Re} \gamma, & v \leq 0 .
\end{array}
$$

Again as $a \rightarrow 2$ the hyperbolic arcs approach the line segment $(-\infty,-1 / 4]$.

The author wishes to thank Professor Roger W. Barnard for many helpful conversations during the writing of this paper.

\section{REFERENCES}

1. I. E. Bazilevič, On a case of integrability in quadratures of the Loewner-Kufarev equation, Mat. Sb. 37 (79) (1955), 471-476. (Russian) MR 17, 356.

2. M. Finkelstein, Growth estimates of convex functions, Proc. Amer. Math. Soc. 18 (1967), 412-418. MR 35 \#5598.

3. T. H. Gronwall, On the distortion in conformal mapping when the second coefficent in the mapping function has an assigned value, Proc. Nat. Acad. Sci. U. S. A. 6 (1920), 300-302.

4. W. K. Hayman, Multivalent functions, Cambridge Tracts in Math. and Math. Phys., no. 48, Cambridge Univ. Press, Cambridge, 1958. MR 21 \#7302.

5. - On functions with positive real part, J. London Math. Soc. 36 (1961), 3548. MR $27 \# 311$. 
6. J. A. Jenkins, On a problem of Gronwall, Ann. of Math. (2) 59 (1954), 490-504. MR 15, 786.

7. C. Pommerenke, Ober nahezu konvexe analytische Funktionen, Arch. Math. (Basel) 16 (1965), 344-347. MR 32 \#7733.

8. —_, On starlike and convex functions, J. London Math. Soc. 37 (1962), 209224. MR 25 \#1279.

9. T. Sheil.Small, On Bazilevič functions, Quart. J. Math. Oxford Ser. (2) 23 (1972), 135-142. MR $45 \# 8847$.

DEPARTMENT OF MATHEMATICS, UNIVERSITY OF KENTUCKY, LEXINGTON, KENTUCKY 40506 